\title{
Reclutamiento y selección a través de las redes sociales Facebook y LinkedIn (análisis preliminar)*
}

\author{
Recruitment and selection through social networks \\ Facebook and LinkedIn (preliminary analysis)
}

\section{Recrutamento e seleção através das redes sociais Facebook e LinkedIn (análise preliminar)}

\author{
Luis Humberto Hernández Díaz** \\ Dra. Ma. del Carmen Liquidano Rodríguez ${ }^{* * *}$ \\ Dra. María de los Ángeles Silva Olvera****
}

\section{RESUMEN}

El objetivo de este trabajo fue identificar y describir las prácticas de reclutamiento y selección de talento humano que aplican las empresas a través de las redes sociales Facebook y LinkedIn, para lo cual se diseńó un estudio no experimental, de corte cuantitativo, de carácter exploratorio, aplicando un cuestionario. Los resultados muestran la tendencia de uso de estas redes sociales en el proceso de reclutamiento y selección de personal, su utilidad con respecto a fuentes y medios convencionales, así como los beneficios que se pueden obtener. Además, se documentan los principales motivos por los que los reclutadores respondientes han descartado a candidatos después de revisar su perfil en estas redes sociales.

Palabras clave: reclutamiento, selección, redes sociales, Facebook, LinkedIn.

\section{ABSTRACT}

The aim of this study was to identify and describe the practices of recruitment and selection of human talent that companies apply through social networks Facebook and LinkedIn, for which a non-experimental study, quantitative court, exploratory study, was designed a questionnaire. The results show the trend of using these social networks in the recruitment and selection of personnel,

* Proyecto alterno de tesis derivado del proyecto de Investigación registrado ante la DGEST (TNM) con el número 5001.13-P. Con el título "Internacionalización de las PYMES en la expatriación y repatriación: influencia del talento humano, la calidad y la cultura emprendedora”, dirigido por la Dra. Ma. del Carmen Liquidano Rodríguez.

** Mexicano, estudiante de la Maestría en Ciencias en Administración, Instituto Tecnológico de Aguascalientes, Aguascalientes, México. Correspondencia con el autor: hernandezdiazhumberto@gmail.com.

*** Mexicana, Maestra en Ciencias en Administración, Doctora en Administración, Instituto Tecnológico de Aguascalientes, Aguascalientes, México. Correspondencia con la autora: maricarmen_inv@yohoo.com.es.

**** Mexicana, Maestra en Ciencias en Administración, Doctora en Administración, Instituto Tecnológico de Aguascalientes, Aguascalientes, México. Correspondencia con la autora: msilvaomx@yahoo.com.mx. 
Reclutamiento y selección a través de las redes sociales Facebook y LinkedIn (análisis preliminar) /

Hernández, et al.

their usefulness with regard to sources and conventional media as well as the benefits to be gained. Furthermore, the main reasons why respondents recruiters have rejected candidates after reviewing your profile on these social networks is documented.

Keywords: recruitment, selection, social networks, Facebook, LinkedIn.

\section{RESUMO}

O objetivo deste estudo foi identificar e descrever as práticas de recrutamento e seleçâo de talento humano que aplicam as empresas através das redes sociais Facebook e LinkedIn, para o qual desenhou-se um estudo não experimental, de natureza quantitativa, de caráter exploratório, aplicando um questionário. Os resultados mostram a tendência do uso destas redes sociais no processo de recrutamento e seleção de pessoal, su utilidade em relação a fontes e médios convencionais, assim como bem como os benefícios a serem obtidos. Além disso, documentam-se os principais motivos pelo qual os recrutadores entrevistados têm descartado candidatos após a análise ao seu perfil nessas redes sociais.

Palavras-chave: recrutamento, seleção, redes sociais, Facebook, LinkedIn.

\section{Introducción}

Hoy en día las organizaciones y empresas tienen la necesidad de identificar e incorporar a aquellas personas que sean consideradas potencialmente idóneas para cubrir alguna vacante. Lo anterior es garantizado a través del uso de técnicas y herramientas estandarizadas que permiten al reclutador atraer, evaluar y contratar al candidato más apto para desempeñar las actividades atribuidas al puesto vacante.

En paralelo, las redes sociales continúan con su crecimiento exponencial en un proceso imparable de adhesión de usuarios, con la incorporación de miles de profesionales través de las muchas plataformas y redes que ya forman parte de una cotidiana actividad social, personal y profesional (Ortega, 2012). Actualmente, las redes sociales tienen una clara orientación social, imperando las relaciones personales y la posibilidad de comunicar, encontrar y compartir información e intereses que enriquecen y proyectan al usuario. Esta proyección de la personalidad, gustos e intereses que manifiesta el usuario, ha permitido a los profesionistas del talento humano utilizar estas plataformas sociales como una herramienta más en los procesos de reclutamiento y selección, permitiéndoles contactar, captar y conocer a candidatos potenciales.

Según Mondy y Noé (2005, en Díaz, 2009:3), el reclutamiento a través del uso de internet se ha vuelto muy popular a nivel mundial, si se considera la velocidad y la amplia cartera de talentos que se pueden obtener por este medio, permitiendo lograr un reclutamiento más eficiente y rentable, tanto para el candidato como para el reclutador. 
De Pablo (2010:3) establece que es en las tecnologías de la información y comunicación, en particular en la web 2.0, donde se desarrolla la nueva forma de comunicación basada en conversaciones colaborativas; es por ello que muchos profesionistas se registran en las redes sociales para buscar empleo, cambiar de empresa o ser promovidos en la que trabajan.

En este mismo sentido, Méndez y García (2009:1) exponen que las personas tienen la necesidad de encontrar un empleo y cada vez menos utilizan los medios tradicionales; de hecho pronostican que en un futuro dejen de hacerlo.

\section{Justificación}

El presente estudio busca generar y dar a conocer conocimiento sólido y sustentado de manera cuantitativa respecto a cómo las organizaciones están utilizando las redes sociales, en específico Facebook y LinkedIn, en el proceso de reclutamiento y selección de talento humano; de esta manera se tendrá información que sea de utilidad para la toma de decisiones de los gestores de talento humano. La investigación aporta valor teórico al documentar y respaldar con el estado del arte, las tendencias de uso de las redes sociales en los procesos de reclutamiento y selección de talento humano, así como se prevé la aportación práctica que permitirá a las empresas aplicar las redes sociales, en particular Facebook y LinkedIn, para que contribuyan a su desarrollo y crecimiento.

Los beneficiarios más importantes de este proyecto son las empresas que aplican o buscan aplicar las redes sociales en las áreas de gestión de talento humano y los candidatos reales y potenciales que usan las redes sociales para ubicarse en mejores puestos de trabajo.

\section{Planteamiento del problema}

Las redes sociales han tenido un éxito sin precedentes en los últimos años, sin embargo Ros-Martín (2009:554) establece su aparición en 1997, momento en que se inaugura la web SixDegrees.com. Para el año 2002, LinkedIn ingresa al mercado de las redes sociales pero con tendencia a los negocios, principalmente para red profesional; permitiendo al usuario crear conexiones directas, subir su currículum vitae, publicar vacantes o colocar algún puesto de trabajo, permitiendo a los reclutadores tener acceso al currículum del usuario. En 2004, la aparición de la red social Facebook oferta servicios tendientes a fortalecer las relaciones sociales a partir de que el usuario desarrolle una lista de contactos con los que se relaciona a través de los servicios de chat, grupos, páginas, muro, fotografías, aplicaciones, juegos, entre otras aplicaciones; que están siendo utilizadas por los empleadores como una herramienta, medio y fuente en el proceso de reclutamiento y selección de talento humano. 
Reclutamiento y selección a través de las redes sociales Facebook y LinkedIn (análisis preliminar) / Hernández, et al.

\section{Objetivo del trabajo}

El objetivo de este trabajo fue identificar y describir las prácticas de reclutamiento y selección de talento humano que aplican las empresas a través de las redes sociales Facebook y LinkedIn. Lo anterior, como análisis preliminar del proyecto de tesis "Reclutamiento y selección a través de las redes sociales Facebook y LinkedIn en la internacionalización de las empresas".

\section{Marco teórico}

\section{Reclutamiento de personal}

Werther (2000), Chiavenato (2000, 2002 y 2012), González y Olivares (2005), Jaramillo (2005), Aguilar (2008) y Lozano (2011) coinciden en sus definiciones en que el reclutamiento de personal es un proceso que conjunta técnicas y procedimientos orientados a la atracción de candidatos potencialmente idóneos para ocupar un puesto vacante en la organización.

\section{Medios de reclutamiento}

Grados Espinoza (2003:8) define los medios de reclutamiento como los mecanismos de difusión que se utilizan para dar a conocer un puesto vacante, ya que muestran gran utilidad por su potencial de difusión, pues penetran y alcanzan distintos ámbitos económicos y sociales; capaces de transmitir información tanto a estructuras socioeconómicas bajas como altas, y a distintos estratos económicos y educativos.

\section{Fuentes de reclutamiento}

Las fuentes de reclutamiento son los lugares de origen donde se puede encontrar de manera concentrada al Talento Humano con un cierto perfil que facilite el cubrir la vacante.

\section{Red social como fuente y medio de reclutamiento}

Aunque el uso de las redes sociales no ha sido considerado ni catalogado dentro de las técnicas o herramientas del proceso de reclutamiento de talento humano, podemos concluir que: Las redes sociales son un medio y una fuente de talento humano.

Son un medio porque cumplen cabalmente con las características señaladas por Grados (2003:8), pues la red social, como Facebook y LinkedIn, es un mecanismo de difusión que permite dar a conocer la vacante; $y$ es una fuente porque permite atraer a candidatos 
que poseen cierto tipo de características, conocimientos o habilidades y que pueden ser localizados fácilmente por su agrupación o pertenencia a determinada escuela, asociación o gremio. Es así como la red social LinkedIn funciona perfectamente como una fuente de reclutamiento, ya que concentra y permite ubicar fácilmente a candidatos con características y conocimientos específicos, que comúnmente buscan pertenecer e integrarse a grupos de su interés.

\section{Selección de personal}

Chiavenato (1996, en Leija, 2012:27) y Lozano (2011:9) coinciden en que la selección de personal es un proceso que lleva a cabo la función de filtro, pues su aplicación permite que solo algunas personas que presentan las características deseadas puedan formar parte de la organización.

\section{Técnicas de selección}

Para el proceso de incorporación de talento humano resulta necesario el uso de técnicas y herramientas de selección, cuya función es permitir al reclutador conocer las características personales, intelectuales y de comportamiento de los candidatos, que al confrontarse a la requisición de personal, se identifique a aquel aspirante que mejor cubra las necesidades del puesto vacante.

Chiavenato (2002:118) agrupa las técnicas de selección en cinco categorías: entrevistas, pruebas de conocimiento o capacidad, pruebas psicométricas, pruebas de personalidad y técnicas de simulación.

\section{Red social}

Las redes sociales son definidas como:

Subrahmanyamam (2008, en Ros-Martín, 2009:555) expone que "las redes sociales son la última herramienta de comunicación en línea que permite a los usuarios crear una entidad pública o semi-pública a través de crear un perfil, lo que le permite ver su cuenta y la de otros usuarios e interactuar con las personas en su red".

Ros-Martín (2009:3) define como servicio de red social en internet a la "plataforma web cuyo fin es la creación de comunidades en línea mediante la representación de las conexiones personales que los usuarios disponen los unos de otros. En estos servicios se puede compartir información mediante la utilización de servicios agregados de mensajería personal, microblogging, publicación de fotografías, formación de grupos de interés, etc.". 
Reclutamiento y selección a través de las redes sociales Facebook y LinkedIn (análisis preliminar) / Hernández, et al.

Méndez y García (2009:1) exponen y definen a las redes virtuales como "el grupo de personas con un interés común que interactúan y se comunican utilizando herramientas web". En este mismo contexto ańaden que el compartir objetivos conlleva a un grado de compromiso que genera una "relación especial" entre ellos.

De Haro (2010:3) define a las redes sociales como "estructuras compuestas por personas u otras entidades humanas, las cuales están conectadas por una o varias relaciones que pueden ser amistad, laboral, intercambios económicos o cualquier otro tema de interés".

\section{Facebook}

Facebook fue fundada en 2004. Su misión es "dar a la gente el poder de compartir y hacer el mundo más abierto y conectado”. Facebook menciona que la gente hace uso de esta red social para mantenerse en contacto con amigos y familiares, para descubrir lo que está pasando en el mundo, y compartir y expresar lo que les importa.

Facebook permite a las personas conectarse con sus amigos, ya sea a través de un juego, una película, compartir su restaurante favorito o escuchar una canción, comentar y compartir artículos o noticias.

\section{LinkedIn}

LinkedIn establece ser la mayor red profesional del mundo con 225 millones de usuarios en más de 200 países y territorios de todo el mundo. Su misión la definen como sencilla: "conectar a los profesionales del mundo para ayudarles a aumentar su productividad y rendimiento. Al unirte a LinkedIn obtienes acceso a personas, empleos, noticias, actualizaciones e información que te ayudarán a destacar en tu campo profesional".

El principal servicio que ofrece LinkedIn es la solución en reclutamiento de talento humano, por lo que se ofrece como una herramienta potente, que permite ampliar las búsquedas más allá de las conexiones que el reclutador posea, para acceder a toda la red LinkedIn y a los 225 millones de usuarios con los que se cuenta. LinkedIn contempla el uso de poderosos filtros que ayudan a concentrarse en los candidatos ideales inmediatamente, sin necesidad de dedicar tiempo a aquellos que no cubren de manera predeterminada el perfil establecido.

\section{Teorías de las redes}

En nuestra cultura resulta común escuchar aquellos dichos en los que se enfatizan las relaciones sociales que el ser humano por naturaleza genera, tal como "Dios los hace 
pero ellos se juntan", "de tal palo tal astilla", "tus amigos son mis amigos", "uno para todos y todos para uno". Estos dichos son el claro ejemplo de la afirmación de Aristóteles: "El hombre es un animal social".

Por lo anterior, podemos suponer que uno de los elementos que ha propiciado el éxito de las redes sociales, es la necesidad del ser humano de socializar y formar parte de "redes", en las que el sujeto comparte determinados gustos, intereses e ideologías. Este tipo de redes se basa principalmente en dos tipos de elementos: "personas" y las "relaciones" que entre estas se generan.

Brea (2011:8) expone en su proyecto de tesis cinco reglas que explican la naturaleza de las redes sociales.

$1^{a}$ Regla: son las personas las que dan forma a sus redes.

2a Regla: la red de la que forman parte las personas da forma a las propias personas.

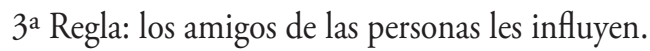

4a Regla: los amigos de los amigos de nuestros amigos también nos influyen.

5a Regla: la red tiene vida propia.

\section{Teoría de los seis grados de separación de Karinthy (1930) y Watts (1998)}

Se le llama "seis grados de separación" o "teoría de los mundos pequeños" a la hipótesis que intenta probar que cualquiera en la Tierra puede estar conectado a cualquier otra persona del planeta, a través de una cadena de conocidos que no tiene más de cinco intermediarios (conectando a ambas personas con sólo seis enlaces) (Brea, 2011:9 y Codina, 2009:8), algo que se ve representado en la popular frase «que chiquito es el mundo». La teoría fue inicialmente propuesta en 1930 por el escritor húngaro Frigyes Karinthy en un cuento llamado Chains.

Según esta teoría, cada persona conoce en promedio, entre amigos, familiares y compañeros de trabajo o escuela, a unas cien personas. Si cada uno de esos amigos o conocidos cercanos se relaciona con otras 100 personas, cualquier individuo puede pasar un recado a 10.000 personas más, tan sólo pidiendo a sus amigos que pasen el mensaje a sus amigos. Estos 10.000 individuos serían contactos de segundo nivel, que un individuo no conoce pero que puede conocer fácilmente pidiendo a sus amigos y familiares que se los presenten, y a los que se suele recurrir para ocupar un puesto de trabajo o realizar una compra. Si esos 10.000 conocen a otros 100, la red ya se ampliaría a 1.000.000 de personas conectadas en un tercer nivel, a 100.000.000 en un cuarto 
Reclutamiento y selección a través de las redes sociales Facebook y LinkedIn (análisis preliminar) /

Hernández, et al.

nivel, a 10.000.000.000 en un quinto nivel y a 1.000.000.000.000 en un sexto nivel. En seis pasos, y con las tecnologías disponibles, se podría enviar un mensaje a cualquier individuo del planeta.

\section{Pluralidad generacional}

Es claro que el auge de las redes sociales se ha visto determinada en la última década, por lo que para conocer su uso como herramienta en el proceso de reclutamiento y selección de personal es necesario tomar conciencia de la pluralidad generacional. Se entiende por generación y/o generacional, al grupo de personas que poseen características comunes, haciendo referencia a una edad determinada o bien a un intervalo determinado de tiempo en la historia. En la demografía la diferencia generacional es una de las variables para la descripción del comportamiento generativo; si bien los grupos generacionales poseen características comunes, sus miembros comúnmente pueden ser muy heterogéneos.

El referirnos a pluralidad generacional responde a individuos que poseen dentro de una misma generación diferentes competencias, habilidades, actitudes, aptitudes y con distinto conocimiento y uso de las tecnologías de la comunicación.

Brea (2011:14) establece de manera cronológica las diferencias generacionales en las siguientes formas:

Baby Boomers (1945-1964). Las personas que se ubican en esta generación son aquellas cuyo nacimiento se sitúa en la posguerra. Sus características que los definen son la disciplina, orden y respeto a los demás. Se caracterizan por su estabilidad laboral, pues muchos de ellos han estado unidos a una empresa o sector desde el comienzo de su actividad profesional y son renuentes al cambio.

Generación X (1965-1981). Son aquellos sujetos cuyo nacimiento se sitúa entre 1965 y 1981, son los hijos de los últimos tradicionalistas de los Baby Boomers. Es una generación que ha sido observadora de los efectos de la globalización y han vivido la evolución de la tecnología desde el surgimiento de la televisión de blanco y negro hasta el Ipad y Smartphone.

Generación Y (1982-1994). Forman parte de dicha generación, aquellas personas cuyos nacimientos se sitúan de 1982 a 1994, junto con la era de la expansión tecnológica. Sus creencias, ideales y valores son muy distintos a quienes conforman la generación X; su crianza se ha visto rodeada de comodidades, independientemente de la clase social. Debido a lo anterior, quienes conforman la generación $Y$ tienden a no apreciar y no valorar la estabilidad del trabajo. Son personas con poco miedo al cambio y pueden 
cambiar rápidamente de trabajo ya que no son leales a sus patrones. Sin embargo, los miembros de esta generación dan valor a las relaciones interpersonales que se crean con compañeros de trabajo. Los ahora jóvenes y miembros de la generación $\mathrm{Y}$, tienden a exhibirse y compartir sus vivencias y pensamientos, por lo que las redes sociales han resultado el escaparate idóneo para esta generación.

Generación Z (1995-Actual). Forman parte de dicha generación los nacidos a partir de 1995 y aunque aún no se pueden establecer diferencias sociológicas respecto a la generación antecesora, se pueden diferenciar en que son la primera generación verdaderamente nativa en la era digital; desde su nacimiento tuvieron acceso a internet, teléfonos inteligentes, computadoras portátiles, entre otro tipo de gadgest; los aún adolecentes son la próxima generación a incorporarse a la vida laboral.

En México, teniendo como fuente el Censo de Población y Vivienda 2010 realizado por el INEGI, el 56,2\% de la población tiene una edad por debajo de los 30 años de edad. Es decir, más de 56 millones de mexicanos son situados en la generación Y y Z.

Estas generaciones ( $\mathrm{Y}$ y Z), valoran y maximizan la utilidad de las redes sociales y en contraste, los Baby Boomers y parte de la generación X, tienen cierta resistencia y desconfianza a participar en las redes sociales, y compartir su vida profesional con el mundo.

\section{Prácticas de reclutamiento y selección de talento humano a través de las redes sociales Facebook y LinkedIn}

Según Mondy y Noé (2005, en Díaz, 2009:3), el reclutamiento a través del uso de internet se ha vuelto muy popular a nivel mundial, si se considera la velocidad y la amplia cartera de talentos que se pueden obtener por este medio, se logra un reclutamiento más eficiente y rentable, tanto para el trabajador como para el reclutador.

Méndez y García (2009:1) exponen que las personas tienen la necesidad de encontrar un empleo, y cada vez menos utilizan los medios tradicionales; de hecho prevén que en un futuro dejen de hacerlo.

De acuerdo con una encuesta que condujo la Society for Human Resources en el Management Career Journal (en Díaz, 2009:11), 80\% de los profesionales en recursos humanos colocan anuncios en internet para encontrar candidatos, y el $96 \%$ de los solicitantes lo usan para encontrar empleos.

Méndez y García (2009:3) consideran que los head-hunters y empresas de selección usan las redes sociales de una forma habitual para realizar sus búsquedas, localizar candidatos e información sobre ellos; por lo anterior, los portales de empleo están 
Reclutamiento y selección a través de las redes sociales Facebook y LinkedIn (análisis preliminar) / Hernández, et al.

redefiniendo su orientación de ser repositorios de oferta y demanda a un concepto de comunidad en la que el candidato intercambia opiniones con empleados de la empresa que publica su oferta y donde puede informarse de manera directa sobre la descripción del puesto.

Teniendo como preámbulo las prácticas anteriormente expuestas, se puede establecer que las redes sociales están reorientando los tradicionales procesos de reclutamiento y selección de talento humano a búsquedas más interactivas, minimizando distancias y cambiando la forma de relacionarse en el mercado laboral.

Las redes sociales permiten a las organizaciones, sin importar su estructura o tamaño, llegar de una manera más ágil a los candidatos, permitiendo que en la búsqueda de talento humano se alcance una mayor cantidad de candidatos, de una manera personalizada e interactiva, reflejándose además, en los indicadores de tiempo, costos, alcance y calidad.

Aunado a lo anterior, Méndez y García (2009:3) consideran que además de los procesos de reclutamiento y selección de talento humano, se verán afectadas aquellas prácticas que se potencian con el intercambio de comunicación y participación, como son el aprendizaje (formación y desarrollo), el desarrollo de relaciones, comunicación organizacional, innovación y trabajo colaborativo.

En este mismo sentido Davidson y Maraist (2011:154) exponen que los cambios de los que está siendo objeto el área de recursos humanos, los ha llevado a usar las redes sociales para reclutar, seleccionar, contratar o rescindir a los individuos; aunque estas prácticas son relativamente nuevas, su uso para estos fines está claramente en aumento, mientras que la investigación sobre estas prácticas es insuficiente.

Brea (2011:21) establece que en los Estados Unidos de América el 80\% de las empresas se apoyan en las redes sociales para sus procesos de reclutamiento y selección, y de estas, el 95\% utiliza LinkedIn.

Díaz Llairó (2009:75) con base en un estudio elaborado por www.employerbraningonline.com, afirma que las plataformas más efectivas para este proceso son:

- Sitio web corporativo $44,6 \%$

- LinkedIn 23,8\%

- Facebook $16,8 \%$

- Twitter $11,9 \%$

- Otros $1,9 \%$

- MySpace $1 \%$ 
De acuerdo con el Centro de Estudios para la Carrera del IE Business de Madrid (en Díaz, 2009:7), el 91\% de las empresas en España utilizan las redes sociales antes de citar a algún candidato a entrevista, por lo que se considera que una mala foto o comentarios con insultos pueden llegar a influir en dejar fuera del proceso de reclutamiento al candidato.

Grensing-Pophal (2012:26) considera que el uso de las redes sociales permite a los profesionales en recursos humanos $(\mathrm{RH})$ dirigirse específicamente a un nicho de candidatos, teniendo como ejemplo la red social LinkedIn, al unirse los gestores de RH a grupos específicos de ciertos intereses. También considera que las redes sociales proporcionan el beneficio de llegar a candidatos pasivos, es decir, aquellos que no se encuentran en búsqueda activa de empleo; sin embargo poseen el perfil y/o conocimientos necesarios para cubrir la vacante; asimismo, la autora seńala la ventaja que Facebook ofrece como una gran herramienta que permite visualizar lo que denomina "marca" del candidato.

Dekay (2009:102) señala como principal atribución de las redes sociales como "promotoras explícitas" de enormes bases de datos de candidatos, fácilmente accesible a los reclutadores. También, deja claro que las personas que cuentan con algún perfil no se pueden considerar como candidatos verdaderamente pasivos; sino que solo tienen interés de ser alertados de ofertas y oportunidades en las cuales puedan mejorar las condiciones de su trabajo actual.

Por lo anterior, son las redes sociales el medio idóneo que propicia y facilita lo que conocemos comúnmente como piratería de personal, que se puede definir como la práctica consistente en persuadir a un candidato que durante el proceso de reclutamiento y selección se encuentra laborando para otra organización, pero que por el grado de conocimientos, especialización, habilidades o aptitudes se considera idóneo para cubrir la vacante.

Hein (2000:3) visualiza que las redes sociales en comparación con las bolsas de trabajo, medios y fuentes convencionales de reclutamiento, permiten llegar a un público que no está buscando trabajo, pero pudieran estar interesados en una oportunidad que les permita una promoción profesional. Asimismo, señala que las redes sociales han permitido romper las barreras geográficas a que comúnmente los medios tradicionales nos sometían.

Rojas (en De Pablo, 2010:2) considera que las redes sociales propician un proceso de reclutamiento rápido, eficiente y poco costoso, afirma que "Buscar, encontrar, seleccionar y reclutar candidatos pasivos es, posiblemente, el proceso con mayor sentido dentro del complejo mercado laboral actual. Pero para hacerlo hay que cambiar tanto de metodología como de mentalidad. Es la única forma de encontrar a los mejores". 
Reclutamiento y selección a través de las redes sociales Facebook y LinkedIn (análisis preliminar) / Hernández, et al.

\section{Implicaciones éticas y aspectos sociales de las prácticas del uso de redes sociales en el reclutamiento y selección de talento humano}

Reclutar y seleccionar debe ser un proceso cauteloso, después de todo, es la única oportunidad que se tiene para escoger a la familia; por lo que el utilizar cualquier método, proceso o herramienta se puede considerar justificado para comprobar la idoneidad del candidato al puesto y a la filosofía de la organización, siempre y cuando se cuente con el consentimiento del candidato. Sin embargo, existen prácticas que han sido limitadas o incluso prohibidas durante este proceso, tal es el caso (en México) de aquellas que menoscaben los derechos humanos y que fomentan la discriminación a partir de decisiones basadas en color, raza, religión, ideología política, origen étnico, orientación sexual, antecedentes penales, uso de narcóticos y drogas e incluso el posible estado de gestación de los candidatos. Este tipo de información ha sido completamente erradicada de los formatos de curriculum vitae y solicitudes de empleo, sin embargo, el uso de las redes sociales en el proceso de reclutamiento y selección da nuevamente al reclutador la facilidad de acceder a este tipo de información.

\section{Metodología}

Se diseñó un estudio no experimental, porque no existe la manipulación de manera deliberada de las variables y son observadas tal y como se presentan en su contexto. Fue exploratorio por tratarse de un tema relativamente nuevo en nuestro contexto. Transversal porque el cuestionamiento de organizaciones y personas se realiza en un solo momento y en un tiempo único y descriptivo por que se buscó documentar el uso y aplicación de las redes sociales en las prácticas de reclutamiento y selección. Se aplicó un cuestionario, con muestreo no probabilístico a cinco empresas que aplican alguna de las fases del proceso de reclutamiento y selección de talento humano en las redes sociales Facebook y/o LinkedIn. Se empleó el análisis de confiabilidad con la mesura de Alfa de Cronbach, y se muestran los resultados en la tabla 1.

Tabla 1. Concentrado de análisis de confiabilidad.

\begin{tabular}{|c|c|c|c|c|}
\hline Variable & $\mathrm{n}=$ & Ítem & Coeficiente Alpha & $\%$ \\
\hline \multicolumn{5}{|c|}{ Redes Sociales } \\
\hline Uso como fuente & 5 & 8 & 0,712 & $71,20 \%$ \\
\hline Uso como medio & 5 & 11 & 0,462 & $46,20 \%$ \\
\hline \multicolumn{5}{|c|}{ Red Social Facebook } \\
\hline Tipo de perfil & 5 & 9 & 0,707 & $70,70 \%$ \\
\hline Uso en reclutamiento & 5 & 8 & 0,734 & $73,40 \%$ \\
\hline
\end{tabular}




\begin{tabular}{|c|c|c|c|c|}
\hline Uso en selección & 5 & 7 & 0,633 & $63,30 \%$ \\
\hline $\begin{array}{c}\text { Viabilidad vs. fuentes y } \\
\text { medios convencionales }\end{array}$ & 5 & 7 & 0,675 & $67,50 \%$ \\
\hline Beneficios & 5 & 3 & 0,429 & $42,90 \%$ \\
\hline Motivo de descarte & 5 & 9 & 0,909 & $90,90 \%$ \\
\hline \multicolumn{7}{|c|}{ Red Social LinkedIn } \\
\hline Tipo de perfil & 5 & 9 & 0,780 & $78,00 \%$ \\
\hline Uso en reclutamiento & 5 & 7 & 0,575 & $57,50 \%$ \\
\hline Uso en selección & 5 & 8 & 0,414 & $41,40 \%$ \\
\hline $\begin{array}{l}\text { Viabilidad vs. fuentes y } \\
\text { medios convencionales }\end{array}$ & 5 & 7 & 0,674 & $67,40 \%$ \\
\hline Beneficios & 5 & 3 & 0,814 & $81,40 \%$ \\
\hline Motivo de descarte & 5 & 9 & 0,581 & $58,10 \%$ \\
\hline
\end{tabular}

Fuente: aportación propia.

\section{Análisis de resultados}

Con este análisis se identificaron cuáles son las prácticas de reclutamiento y selección de talento humano que aplican las empresas a través de las redes sociales Facebook y LinkedIn, las cuales se resumen en las gráficas de la 1 a la 17.

\section{Datos generales y perfil demográfico del gestor de talento humano (reclutador)}

Gráfica 1. Resumen de resultados datos generales y perfil demográfico del gestor de talento humano.

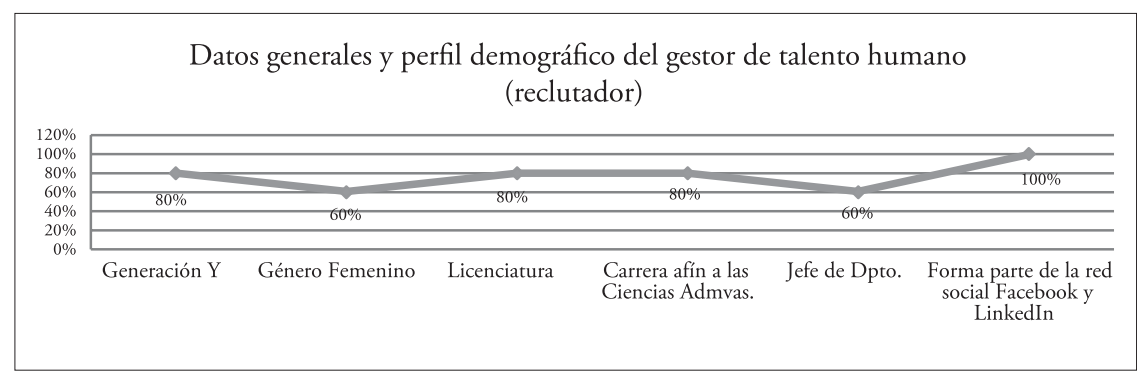

Fuente: aportación propia.

El 80\% de los respondientes de la gráfica 1, forman parte de la generación $Y$, de los cuales el $60 \%$ son del sexo femenino, $80 \%$ del total de los encuestados poseen formación de licenciatura en alguna carrera afín a las ciencias administrativas. El 100\% manifiesta formar parte de la red social Facebook y LinkedIn. 
Reclutamiento y selección a través de las redes sociales Facebook y LinkedIn (análisis preliminar) / Hernández, et al.

\section{Datos generales de la empresa}

Gráfica 2. Resumen de resultados datos generales de la empresa.

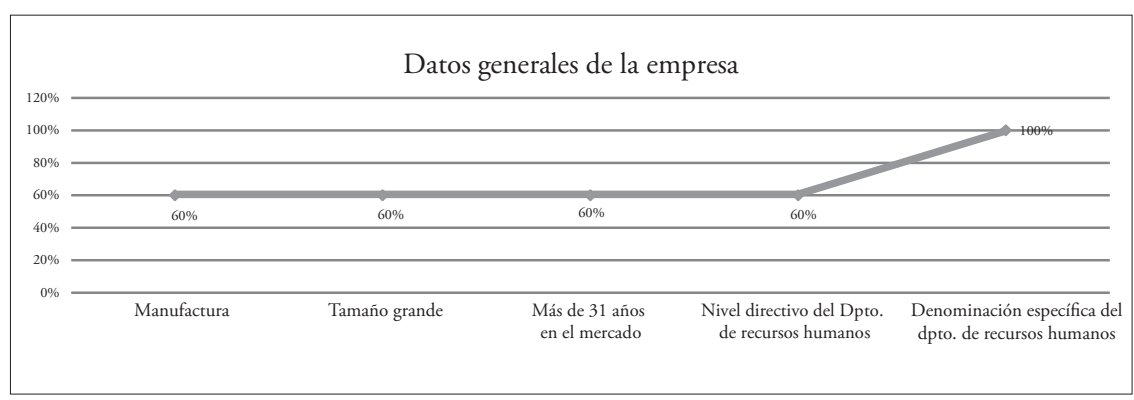

Fuente: aportación propia.

En la gráfica 2, el 60\% de los respondientes laboran para empresas que tienen como giro la manufactura, el mismo porcentaje se mantiene para las organizaciones que según el número de trabajadores son catalogadas grandes, y que cuentan con más de 31 ańos de su constitución. $60 \%$ de los respondientes mantienen el departamento de recursos humanos con nivel jerárquico de dirección. El 100\% de los encuestados precisan que dentro de la organización el departamento de recursos humanos mantiene una denominación específica.

\section{Prácticas de reclutamiento y selección de personal a través de las redes sociales}

Gráfica 3. Facebook y LinkedIn y su uso en el proceso de reclutamiento.

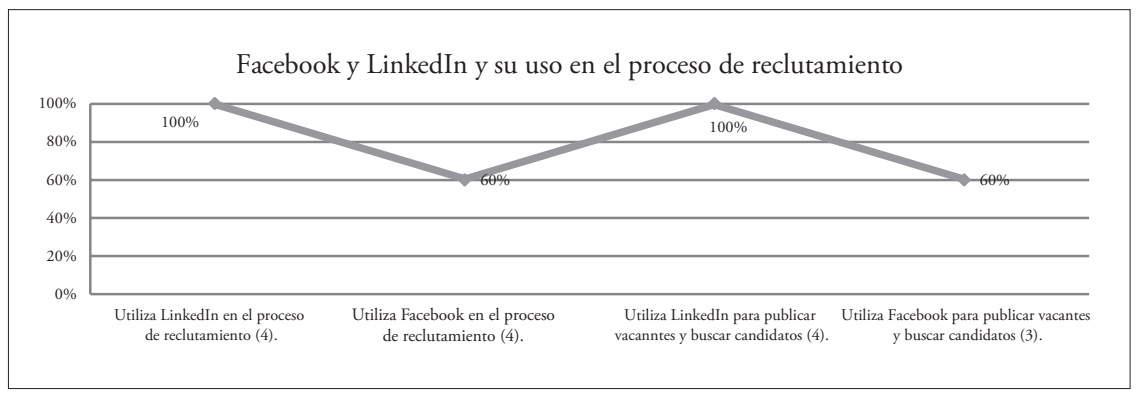

(4) La mayoría de las veces, (3) regularmente.

Fuente: aportación propia. 
De los respondientes de la gráfica 3, el 100\% manifiesta utilizar la red social LinkedIn en el proceso de reclutamiento, y el $60 \%$ la red social Facebook en este mismo proceso. LinkedIn es utilizada por el 100\% para publicar vacantes y buscar candidatos, mientras Facebook solo el $60 \%$.

Gráfica 4. Frecuencia de uso de Facebook para reclutar según el perfil del puesto vacante.

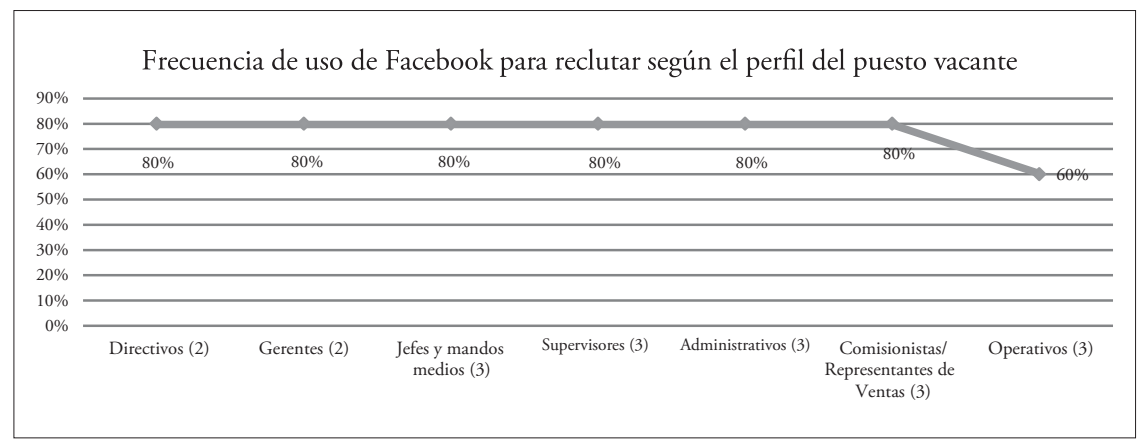

(3) Regularmente, (2) rara vez.

Fuente: aportación propia.

El $80 \%$ de los respondientes de la gráfica 4, coinciden que regularmente se utiliza Facebook para reclutar candidatos para el área administrativa, supervisores, mandos medios y ventas; para otros perfiles rara vez se utiliza la mencionada red social.

Gráfica 5. Frecuencia de uso de LinkedIn para reclutar según el perfil del puesto vacante.

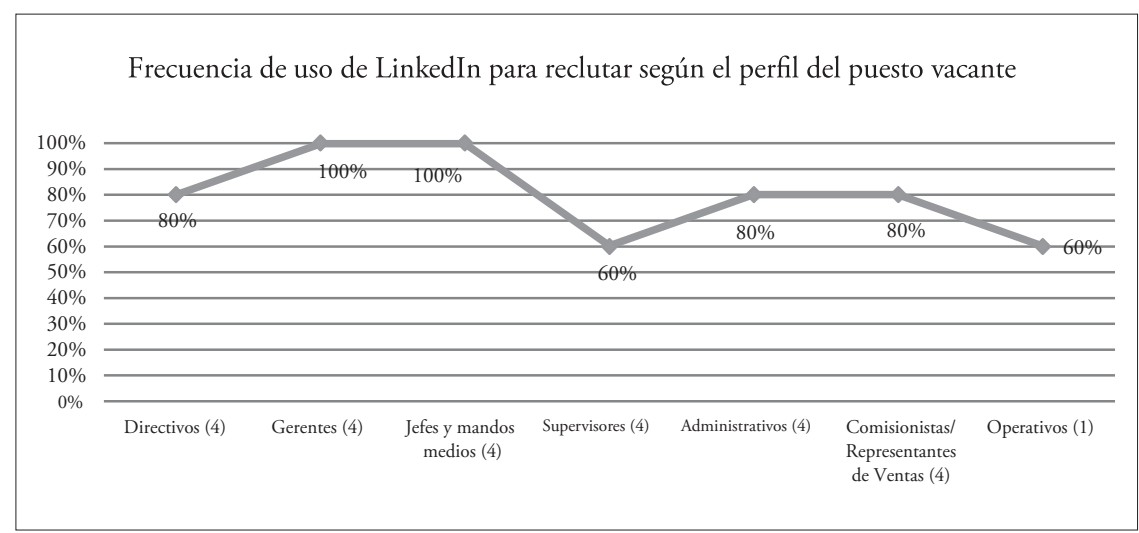

(4) La mayoría de las veces, (1) nunca.

Fuente: aportación propia.

El 100\% de los encuestados de la gráfica 5, coinciden en utilizar la mayoría de las veces la red social LinkedIn para reclutar candidatos a ocupar las vacantes en puestos 
Reclutamiento y selección a través de las redes sociales Facebook y LinkedIn (análisis preliminar) / Hernández, et al.

gerenciales, jefaturas y mandos medios. El 80\% coinciden en utilizar la mayoría de las veces esta misma red para reclutar directivos, administrativos y personal de ventas. En contraste, el 60\% manifiesta nunca utilizar LinkedIn para reclutar personal operativo.

Gráfica 6. Frecuencia de uso de Facebook vs. intención de uso en el proceso de reclutamiento.

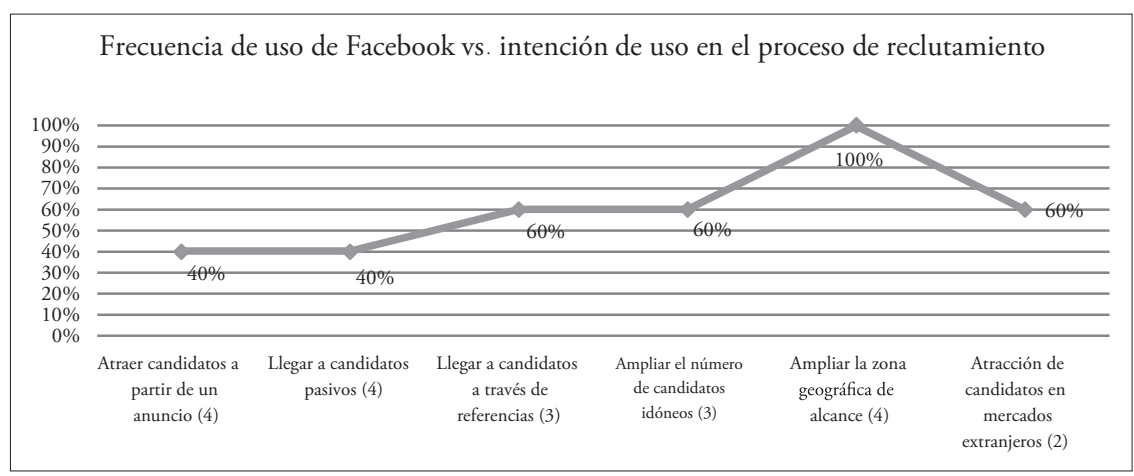

(4) La mayoría de las veces, (3) regularmente, (2) rara vez.

Fuente: aportación propia.

El 100\% de los respondientes de la gráfica 6, utilizan la mayoría de las veces la red social Facebook para ampliar la zona geográfica de reclutamiento, $40 \%$ la utilizan en la mayoría de las veces para publicar anuncios de vacantes y contactar candidatos pasivos. $60 \%$ regularmente pretenden a través del uso de Facebook ampliar el número de candidatos potencialmente idóneos en el proceso de reclutamiento de talento humano.

Gráfica 7. Frecuencia de uso de LinkedIn vs. intención de uso en el proceso de reclutamiento.

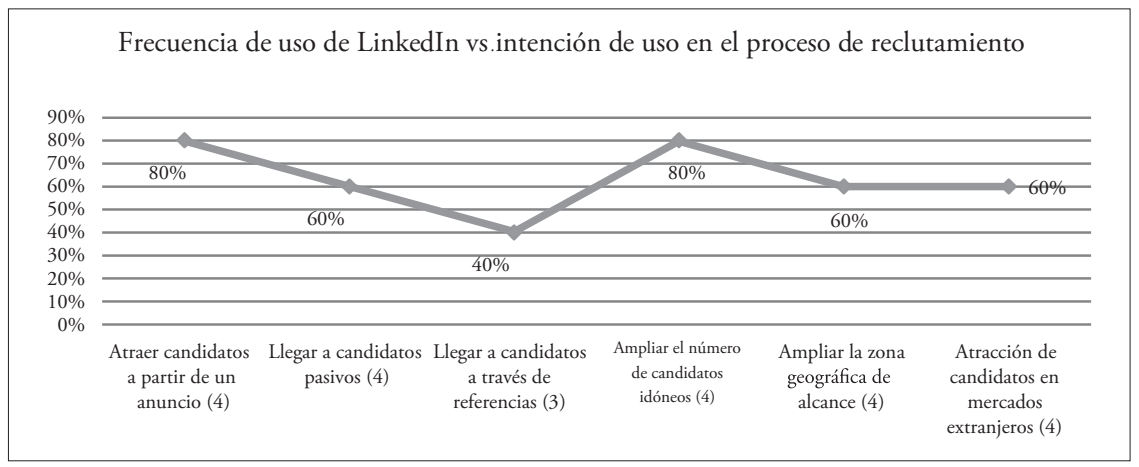

(4) La mayoría de las veces, (3) regularmente.

Fuente: aportación propia. 
El $80 \%$ de los encuestados en la gráfica 7 , mencionan que en la mayoría de las veces utilizan la red social LinkedIn para atraer candidatos a partir de un anuncio y ampliar el número de candidatos. El 60\% coinciden en que la mayoría de las veces usan esta red con la intención de llegar a candidatos pasivos, ampliar la zona geográfica, así como atraer candidatos de mercados extranjeros.

Gráfica 8. Frecuencia de uso de Facebook en el proceso de selección de talento humano.

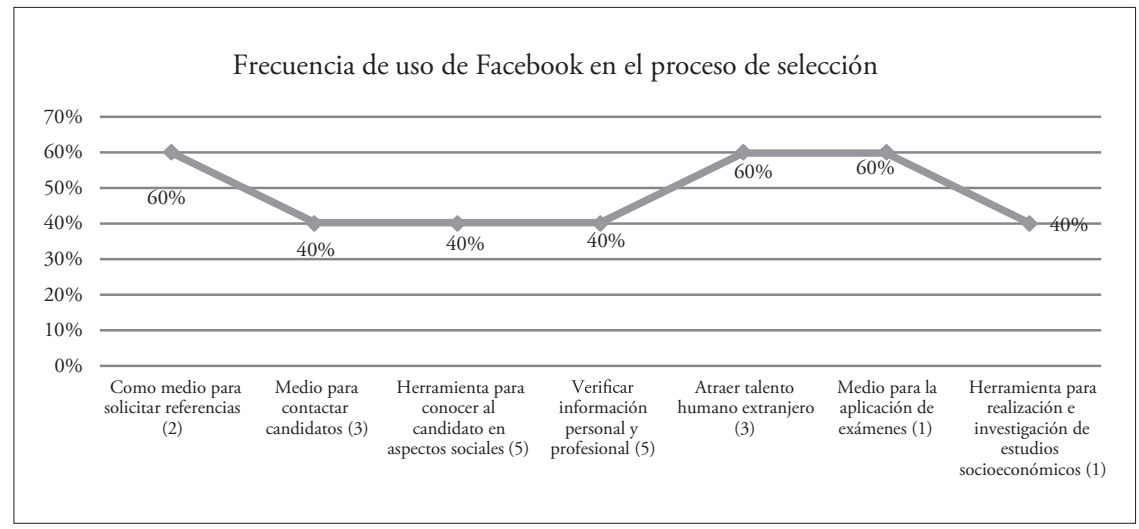

(5) Siempre, (3) regularmente, (2) rara vez, (1) nunca.

Fuente: aportación propia.

Con respecto al uso de Facebook en el proceso de reclutamiento de personal de la gráfica 8 , el 40\% de los respondientes coinciden en siempre utilizar la red social para verificar información personal y profesional, así como herramienta que permite conocer al candidato en aspectos sociales.

Gráfica 9. Frecuencia de uso de LinkedIn en el proceso de selección de talento humano.

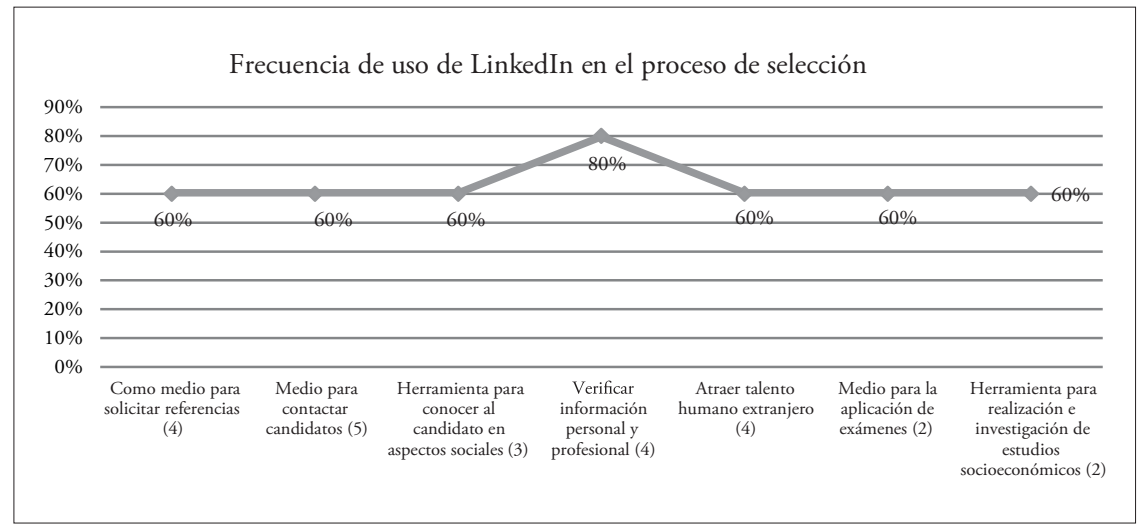

(5) Siempre, (4) la mayoría de las veces, (3) regularmente, (2) rara vez.

Fuente: aportación propia. 
Reclutamiento y selección a través de las redes sociales Facebook y LinkedIn (análisis preliminar) / Hernández, et al.

En la gráfica 9 la red social LinkedIn es utilizada en el proceso de reclutamiento de personal, siempre por el 60\% de los respondientes para poder contactar candidatos, y el $80 \%$ para la mayoría de las veces verificar información personal y profesional.

Gráfica 10. Frecuencia de uso de Facebook en el proceso de selección de talento humano.

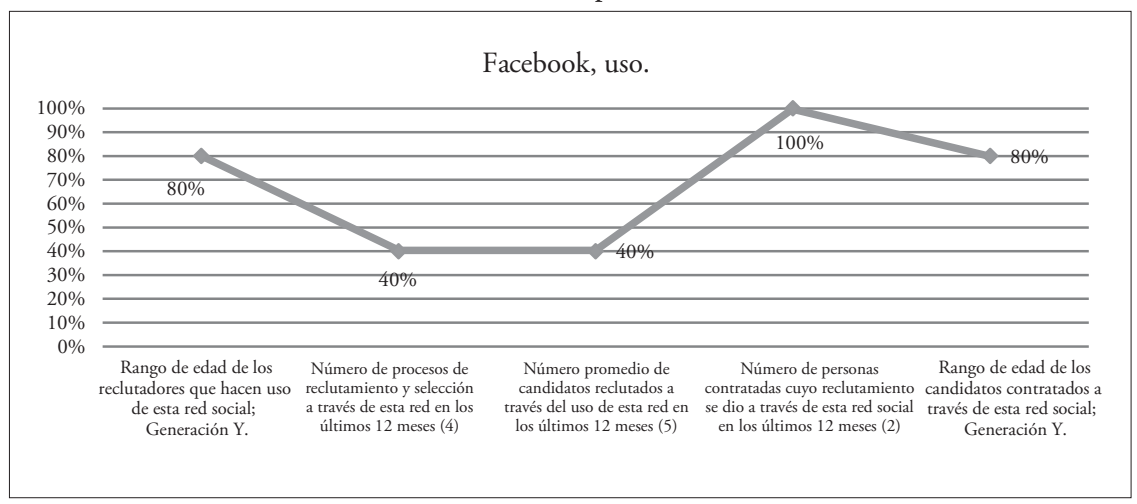

(5) Más de 50, (4) entre 21 y 50, (2) entre 1 y 10.

Fuente: aportación propia.

De los reclutadores que utilizan Facebook en la gráfica 10, para desarrollar alguna de las fases del proceso de reclutamiento y selección de personal, el $80 \%$ se sitúa en base a su edad en la generación Y; el 40\% coincide en que durante los últimos 12 meses han realizado entre 21 y 50 procesos de reclutamiento a través de esta red, y se han reclutado más de 50 candidatos. El $80 \%$ de los contratados que fueron reclutados y/o seleccionados a través de la red social Facebook, también cuentan con edad que los sitúa como miembros de la generación Y (entre 20 y 32 años de edad).

Gráfica 11. Frecuencia de uso de LinkedIn en el proceso de selección de talento humano.

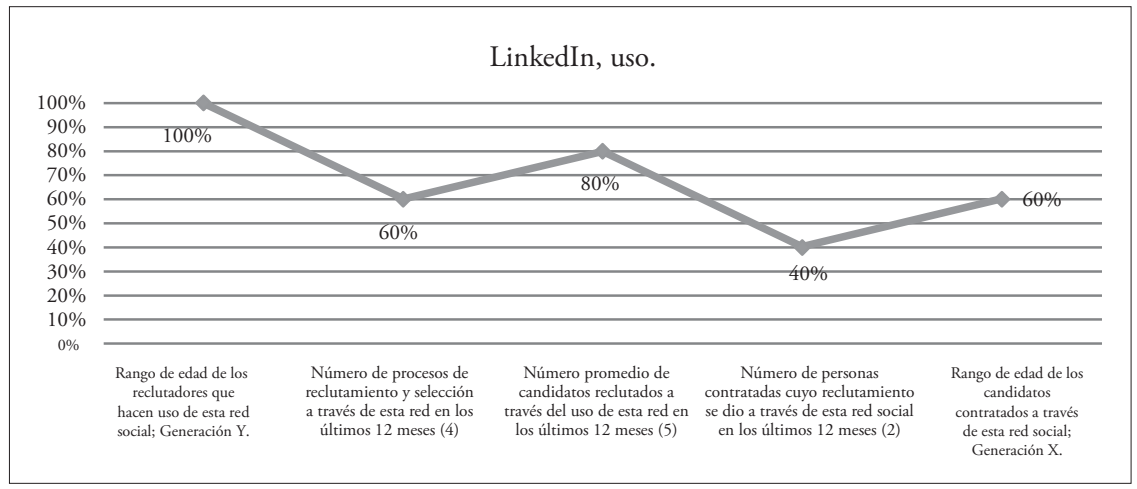

(5) Más de 50, (4) entre 21 y 50.

Fuente: aportación propia. 
El 100\% de los reclutadores que utilizan la red social LinkedIn en la gráfica 11, tienen un rango de edad que los ubica como miembros de la generación Y. El 60\% coinciden en que durante los últimos 12 meses han realizado más de 50 procesos de reclutamiento y selección a través de LinkedIn; el $80 \%$ ha reclutado en este mismo periodo a más de 50 candidatos; de los cuales, el 40\% de los respondientes coinciden que se han contratado entre 21 y 50 candidatos. Dentro de la generación X se ubican las edades de las personas contratadas a través de LinkedIn.

Gráfica 12. Percepción de utilidad de Facebook respecto a las fuentes y medios convencionales en el proceso de reclutamiento y selección de talento humano.

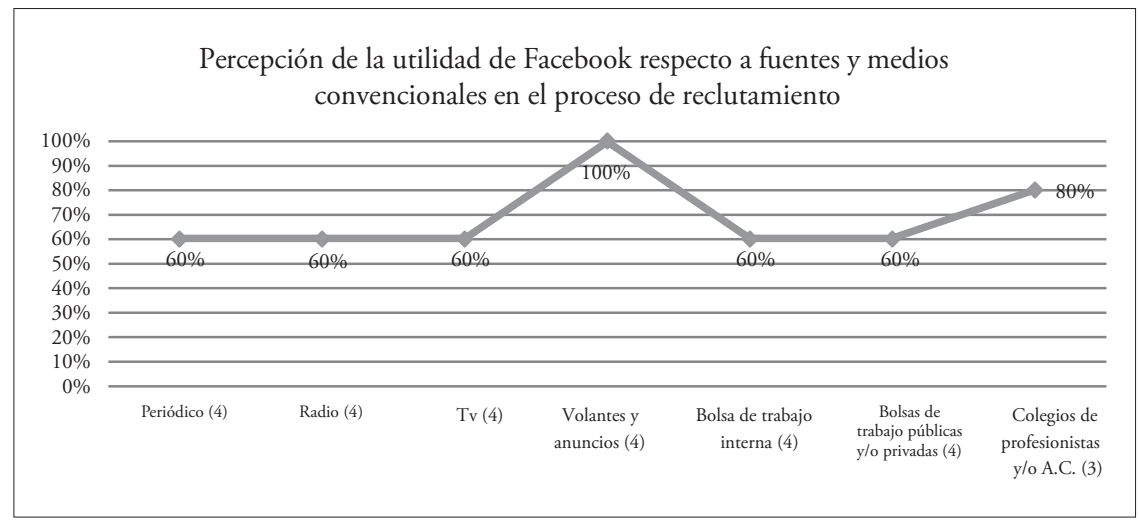

(4) Más útil, (3) igual de útil.

Fuente: aportación propia.

El 100\% de los respondientes para la gráfica 12, coinciden que utilizar Facebook en el proceso de reclutamiento y selección de talento humano es más útil que respecto a volantes y anuncios, y el $60 \%$ coincide en que esta red social es más útil que el periódico, radio, tv, bolsa interna de trabajo y bolsas externas públicas y privadas. 
Reclutamiento y selección a través de las redes sociales Facebook y LinkedIn (análisis preliminar) / Hernández, et al.

Gráfica 13. Percepción de utilidad de LinkedIn respecto a las fuentes y medios convencionales en el proceso de reclutamiento y selección de talento humano.

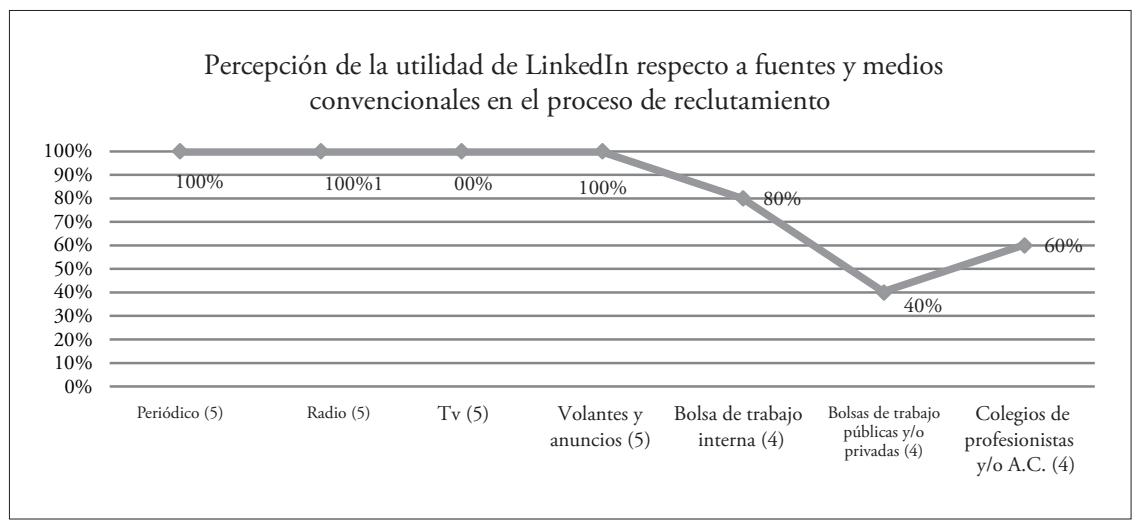

(5) Mucho más útil, (4) más útil.

Fuente: aportación propia.

El 100\% de los respondientes en la gráfica 13, coinciden que utilizar LinkedIn en el proceso de reclutamiento y selección de talento humano es mucho más útil que respecto a periódico, radio, volantes y anuncios.

Gráfica 14. Grado de beneficio al usar Facebook en el proceso de reclutamiento y selección de talento humano en comparación con las fuentes y medios convencionales.

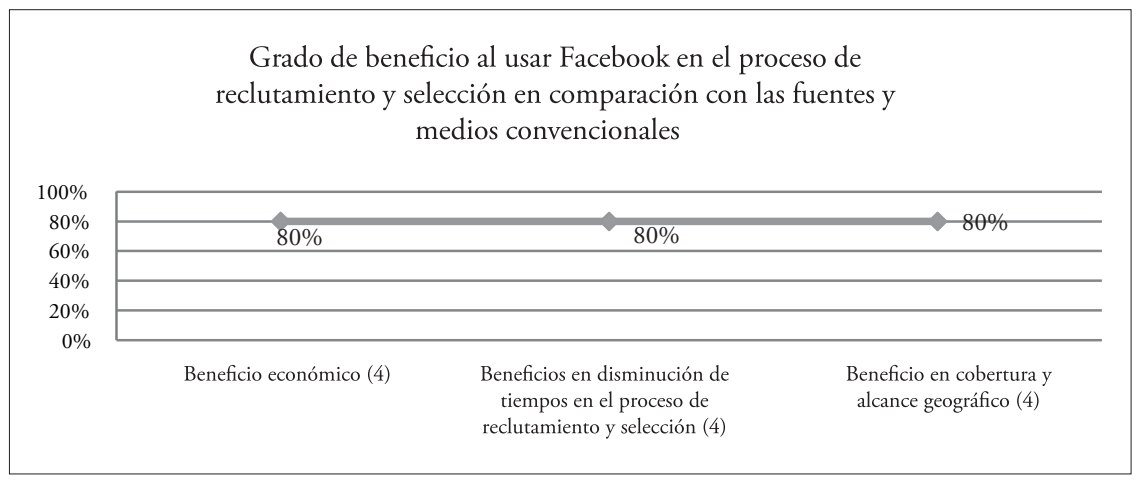

(4) La mayoría de las veces.

Fuente: aportación propia.

Coinciden el $80 \%$ de los respondientes en la gráfica 14, que la mayoría de las veces al utilizar Facebook en el proceso de reclutamiento y selección se generan beneficios económicos, se disminuyen tiempos, existe mayor cobertura y alcance geográfico respecto a los medios convencionales de reclutamiento. 
Gráfica 15. Grado de beneficio al usar LinkedIn en el proceso de reclutamiento y selección de talento humano en comparación con las fuentes y medios convencionales. (5) Siempre.

\begin{tabular}{|c|c|c|c|}
\hline \multirow{5}{*}{$\begin{array}{r}100 \% \\
80 \% \\
60 \% \\
40 \% \\
20 \% \\
0 \%\end{array}$} & \multicolumn{3}{|c|}{$\begin{array}{l}\text { Grado de beneficio al usar LinkedIn en el proceso de } \\
\text { reclutamiento y selección en comparación con las fuentes y } \\
\text { medios convencionales }\end{array}$} \\
\hline & \multicolumn{2}{|l|}{$100 \%$} & $-100 \%$ \\
\hline & & & \\
\hline & & & \\
\hline & Beneficio económico (5) & $\begin{array}{l}\text { Beneficios en disminución de } \\
\text { tiempos en el proceso de } \\
\text { reclutumiento y selección (5) }\end{array}$ & $\begin{array}{l}\text { Beneficio en cobertura y } \\
\text { alcance geográfico (5) }\end{array}$ \\
\hline
\end{tabular}

Fuente: aportación propia.

Coinciden el 100\% de los respondientes en la gráfica 15, que siempre que se utiliza LinkedIn en el proceso de reclutamiento y selección, se generan beneficios económicos, disminución de tiempos y mayor cobertura y alcance geográfico respecto a los medios convencionales de reclutamiento.

Gráfica 16. Motivos por los que se ha descartado a candidatos después de revisar su perfil en Facebook.

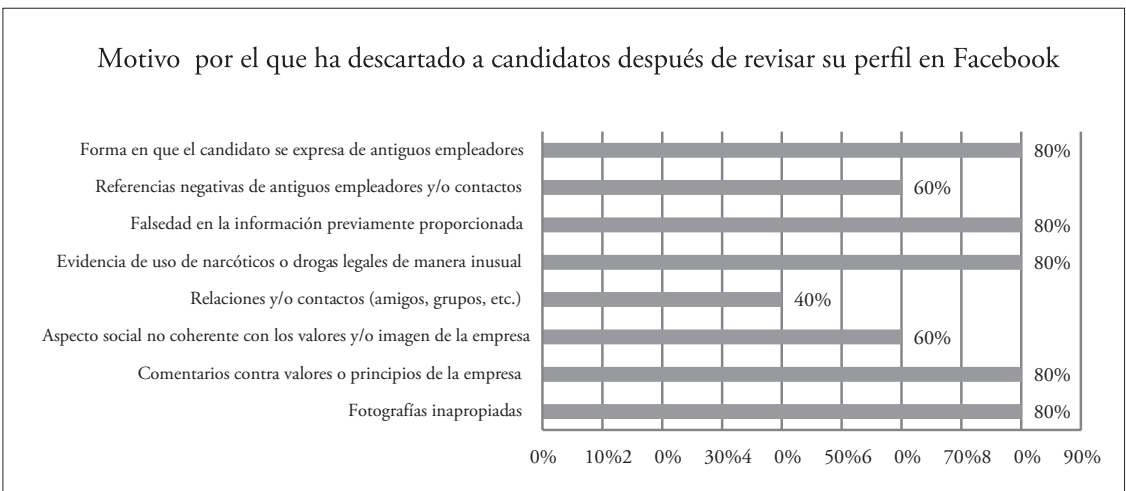

Fuente: aportación propia.

El 80\% de los encuestados de la gráfica 16, admiten haber descartado candidatos después de revisar su perfil en Facebook, por expresarse de manera que se considera inadecuada de anteriores empleadores, por falsedad de información, por evidenciar el uso de narcóticos o drogas legales de manera poco usual, cuando el candidato realizó comentarios que se consideran en contra de los valores o principios de la organización, así como mantener fotografías que se valoraron como inapropiadas por el reclutador. 
Reclutamiento y selección a través de las redes sociales Facebook y LinkedIn (análisis preliminar) / Hernández, et al.

Gráfica 17. Motivos por los que se ha descartado a candidatos después de revisar su perfil en LinkedIn.

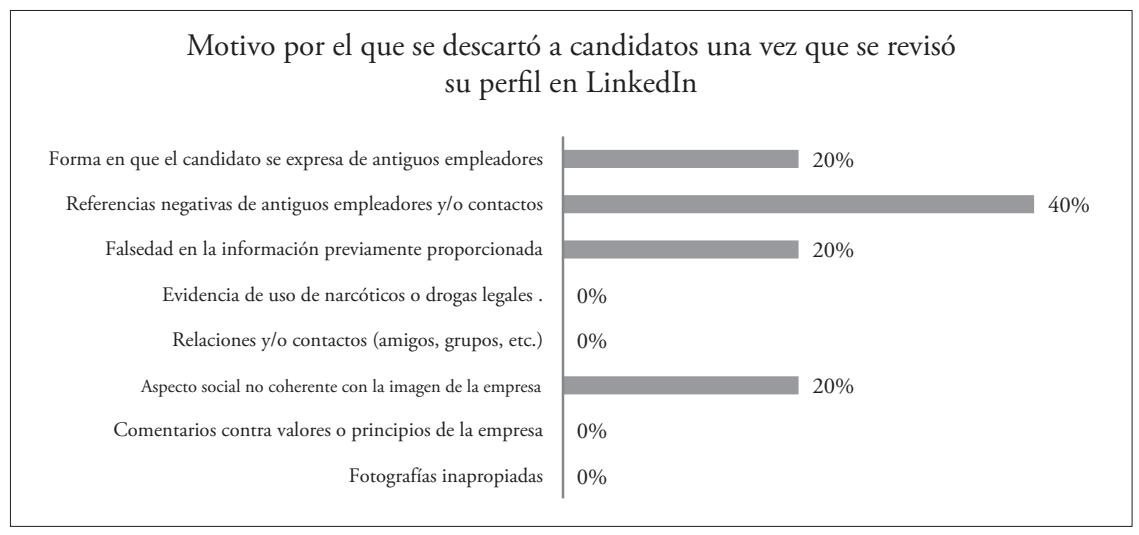

Fuente: aportación propia.

El 40\% de los encuestados en la gráfica 17, admiten haber descartado candidatos después de revisar su perfil en LinkedIn por referencias negativas de antiguos empleadores; no se descartaron candidatos por motivos de evidenciar uso de narcóticos o drogas, por las relaciones o contactos que posee el candidato, comentarios o fotografías que se pudieran llegar a considerar inapropiadas.

\section{Conclusiones}

Se identificaron y describieron las prácticas de reclutamiento y selección de talento humano que aplican las empresas a través de las redes sociales Facebook y LinkedIn. En este sentido, se observa que es afirmativo el supuesto que se encuentra en incremento el uso de estas redes sociales en las diferentes etapas del proceso de reclutamiento y selección de personal; siendo los principales candidatos reclutados en LinkedIn aquellos para cubrir vacantes directivas, gerencias, jefaturas y técnicos especializados, mientras en Facebook principalmente se reclutan, jefes, supervisores, mandos medios y administrativos.

Dentro de los principales beneficios documentados se destaca el que los procesos de reclutamiento y selección a través de las redes sociales, resultan más económicos y permiten un mayor alcance, así como, la extraordinaria posibilidad de llegar a candidatos pasivos.

Facebook resulta idóneo principalmente para verificar el contexto social, cultural y económico del candidato; mientras LinkedIn muestra al candidato en el ámbito profesional y académico. 
En este mismo sentido, se establece que tanto reclutadores y candidatos que utilizan las redes sociales pertenecen a la generación X e Y; por lo que resulta predecible que las redes sociales pasarán a formar parte en el corto plazo como fuente, medio, herramienta y canal idóneo y preferido por los reclutadores; respaldando este supuesto la percepción de utilidad que los respondientes mencionan de las redes sociales respecto a las fuentes y medios convencionales.

También se muestra evidencia empírica que en mayor medida, mediante el uso de Facebook en el proceso de reclutamiento y selección de personal, se obtiene información del candidato que puede ser considerada como "sensible", y que no es posible obtener de alguna otra forma sin caer en actos discriminatorios.

Los anteriores hallazgos refuerzan la viabilidad de incrementar el número de encuestados y vincular el uso de las redes sociales Facebook y LinkedIn con respecto al grado de internacionalización de las empresas en el proyecto de tesis del cual se desprende este trabajo.

\section{Bibliografía}

Aguilar F., Maricela (2008). Reclutamiento y selección de personal: herramienta clave para el desarrollo de la pymes. Universidad Veracruzana. Monografía para obtener el título de Licenciado en Administración. Xalapa-Enríquez, Veracruz, México.

Brea R., Jesús Ramón (2011). Redes sociales como herramienta de reclutamiento y selección. Proyecto Máster, Escuela de Negocios Novacaixagalicia.

Chiavenato, Idalberto (2000). Administración de recursos humanos. México, DF. McGraw Hill Interamericana, S.A.

Chiavenato, Idalberto (2002). Gestión del talento humano. Bogotá, Colombia. McGraw Hill Interamericana, S.A.

Codina, Luis (2009). "Ciencia 2.0: redes sociales y aplicaciones en línea para académicos" [on line]. Hipertext.net, núm. 7, 2009. http://www.upf.edu/hipertextnet/numero-7/ciencia-2-0.html

Davison Kristl, Maraist Catherine (2011). Frien dor foe? The promise and pitfalls of using social networking sites for HR decisions. Published online: 4 May 2011, Springer Science+Business Media, LLC 2011.

De Haro, Juan José (2010). Redes sociales en educación. Colegio Amor de Dios. Barcelona, España.

De Pablo, S. (2010). "El impacto 2.0 en la búsqueda y selección de profesionales con talento". Capital Humano, No. 248. 
Reclutamiento y selección a través de las redes sociales Facebook y LinkedIn (análisis preliminar) / Hernández, et al.

Dekay, Sam (2009). Are business-oriented social networking web site useful resources for locating passive job seekers? Results of a recent study. The Bank of New York Mellon Corporation and St. John's University.

Díaz, Carlos (2009). El reclutamiento de talentos por medio de las redes sociales en empresas mexicanas: ¿Realidad o ficción? Visto en liga:

http://www.academia.edu/1927979/_El_Reclutamiento_de_Talentos_por_ Medio_de_las_Redes_Sociales_en_Empresas_Mexicanas_Realidad_o_Ficcion_

Díaz Llairó, Amparo (2011). El talento está en la red. Ed. Lid. España. 288.

García del Castillo, José A. (2013). "Adicciones tecnológicas: el auge de las redes sociales". Instituto de Investigación de Drogadependencias (INID). Health and Addictions 2013, Vol. 13, No.1, 5-14.

Grensing-Pophal, Lin (2012). Social recruitment; LinkedIn, Facebook and Twitter are useful tools for finding new employees, but be cautious. Credit Union Management.

Hein, Rich (2009). Tips for finding top IT talenton LinkedIn. Visto en: www.cio.com (Lectura 06)

Jaramillo N., Olga Lucía (2005). "Gestión del talento humano en la micro, pequeña y mediana empresa vinculada al programa Expopyme de la Universidad del Norte en los sectores de confecciones y alimentos". Pensamiento y Gestión, no. 18, Barranquilla, Colombia. ISSN 1657-6276.

Leija F., Alejandra (2012). Prácticas de administración de capital humano y el desempeño organizacional en las pymes de servicios en Aguascalientes. Instituto Tecnológico de Aguascalientes. Tesis para obtener el grado de Maestría. Aguascalientes, México.

Lozano, Raymundo (2011). Aportaciones a la tesis doctoral: El reclutamiento, selección, contratación y desarrollo del docente bajo el modelo educativo basado en competencias como propuesta para las universidades politécnicas de México.

Méndez D., Elena y García P., José M. (2009). "Las redes virtuales: nuevas formas de encontrar trabajo y candidatos". Capital Humano N ${ }^{\circ} 237$. Localizado en http:// www.capitalhumano.es/ver_detalleArt.asp?idArt=58987\&action=imprimir

Ortega, Andrés (2012). 5 aspectos organizativos que delimitan el reclutamiento 2.0. Visto en: https://humanbeingelblogdeandresortega.wordpress.com/category/ people-management-human-being/recruitment/.

Ros-Marín, Marcos (2009). "Evolución de los servicios de redes sociales en internet”. El profesional de la información. Septiembre-Octubre, v. 18, n. 5, pp. 552-557. 


\section{Cómo citar este artículo:}

Hernández, L.; Liquidano, M. y Silva, M. (2014). "Reclutamiento y selección a través de las redes sociales Facebook y LinkedIn (análisis preliminar)", Oikos No 38, 37 - 61, Escuela de Administración y Economía, Universidad Católica Silva Henríquez (UCSH), Santiago de Chile.

[http://ediciones.ucsh.cl/revistas.php]

Fecha de recepción: 01 / 10 / 2014

Fecha de aceptación: 26 / 12 / 2014 
